\title{
ON PROPER (STRONG) RAINBOW CONNECTION OF GRAPHS
}

\author{
Hui Jiang, Wenjing Li \\ Center for Combinatorics and LPMC \\ Nankai University, Tianjin 300071, China \\ e-mail: jhuink@163.com \\ liwenjing610@mail.nankai.edu.cn \\ Xueliang Li \\ Center for Combinatorics and LPMC \\ Nankai University, Tianjin 300071, China \\ and \\ School of Mathematics and Statistics \\ Qinghai Normal University, Xining, Qinghai 810008, China \\ e-mail: lxl@nankai.edu.cn \\ AND \\ Colton Magnant \\ Department of Mathematics \\ Clayton State University, Morrow, GA, USA \\ e-mail: dr.colton.magnant@gmail.com
}

\begin{abstract}
A path in an edge-colored graph $G$ is called a rainbow path if no two edges on the path have the same color. The graph $G$ is called rainbow connected if between every pair of distinct vertices of $G$, there is a rainbow path. Recently, Johnson et al. considered this concept with the additional requirement that the coloring of $G$ is proper. The proper rainbow connection number of $G$, denoted by $\operatorname{prc}(G)$, is the minimum number of colors needed to properly color the edges of $G$ so that $G$ is rainbow connected. Similarly, the proper strong rainbow connection number of $G$, denoted by $\operatorname{psrc}(G)$, is the minimum number of colors needed to properly color the edges of $G$ such that for any two distinct vertices of $G$, there is a rainbow geodesic (shortest path) connecting them. In this paper, we characterize those graphs with proper rainbow connection numbers equal to the size or within 1 of the
\end{abstract}


size. Moreover, we completely solve a question proposed by Johnson et al. by proving that if $G=K_{p_{1}} \square \cdots \square K_{p_{n}}$, where $n \geq 1$, and $p_{1}, \ldots, p_{n}>1$ are integers, then $\operatorname{prc}(G)=\operatorname{psrc}(G)=\chi^{\prime}(G)$, where $\chi^{\prime}(G)$ denotes the chromatic index of $G$. Finally, we investigate some sufficient conditions for a graph $G$ to satisfy $\operatorname{prc}(G)=r c(G)$, and make some slightly positive progress by using a relation between $\operatorname{rc}(G)$ and the girth of the graph.

Keywords: proper (strong) rainbow connection number, Cartesian product, chromatic index.

2010 Mathematics Subject Classification: 05C15, 05C40, 05C75.

\section{REFERENCES}

[1] J.A. Bondy and U.S.R. Murty, Graph Theory (Springer, 2008).

[2] G. Chartrand, G.L. Johns, K.A. McKeon and P. Zhang, Rainbow connection in graphs, Math. Bohem. 133 (2008) 85-98.

[3] P. Johnson, E. Jones, K. Kumwenda, R. Matzke and S. Bau, Rainbow connectivity in some Cayley graphs, Australas. J. Combin. 71 (2018) 381-393.

[4] X. Li, Y. Shi and Y. Sun, Rainbow connections of graphs: A survey, Graphs Combin. 29 (2013) 1-38.

doi:10.1007/s00373-012-1243-2

[5] X. Li and Y. Sun, An updated survey on rainbow connections of graphs - a dynamic survey, Theory Appl. Graphs 0(1) (2017), Art. 3. doi:10.20429/tag.2017.000103

[6] X. Li and Y. Sun, Rainbow Connections of Graphs (Springer-Verlag, New York, 2012).

Received 23 July 2018

Revised 9 January 2019

Accepted 9 January 2019 